\title{
Future Neutrino Experiments, DUNE and T2HK
}

\author{
Jaehoon $\mathbf{Y u}^{1}$ \\ The University of Texas at Arlington \\ Department of Physics, 502 Yates St., University of Texas, Arlington, TX 76019, USA \\ E-mail: jaehoonyu@uta.edu \\ Neutrinos are massless charge neutral leptons in the Standard Model. The discovery and the solid \\ confirmation of their flavor oscillation, however, necessarily require them to be massive since this \\ is caused by the fact that the neutrino mass eigenstates differ from that of the flavor. This \\ necessitates the Standard Model to be significantly modified or a new paradigm to describe the \\ fundamental constituents of matter. To accomplish this goal, it is essential for experiments to \\ measure precisely various oscillation parameters, including the mixing angles, to determine the \\ mass hierarchy between the three mass eigenstates and to determine the CP phase $\left(\delta_{\mathrm{CP}}\right)$. This \\ presentation covers two large scale future neutrino experiments, the Deep Underground Neutrino \\ Experiment (DUNE) and the Tokai-to-Hyper Kamiokande (T2HK) experiments which along with \\ the high intensity neutrino beam line will help accomplish these goals. The design and \\ technologies of these two experiments, their physics capabilities as well as their schedule are \\ presented.
}

The 39th International Conference on High Energy Physics (ICHEP2018)

4-11 July, 2018

Seoul, Korea

\section{${ }^{1}$ Speaker}




\section{Introduction}

Three most representative discoveries relevant to the basic fundamental particle map of the Standard Model [1] in the past quarter century arguably are the observation of top quark at Fermilab announced in 1995 [2], the discovery of Higgs like scalar boson at CERN announced in 2012 [3] and neutrino flavor oscillation discovery which was confirmed throughout the '90s and the 2000's, reviewed in Ref. [4]. Of these three, the first confirms the Standard Model by completing the quark sector map and the second, newly discovered Higgs-like boson seems to increasingly behave like the one predicted by the Standard Model while the third goes against it. Since neutrino oscillation occurs due to the fact that the flavor eigenstate differs from the mass eigenstate, the fact that the neutrinos undergo the flavor oscillation means that neutrinos have mass. The Standard Model, however, does not prescribe masses to neutrinos, is broken in the neutrino sector and thus require modifications or a completely new paradigm of theory is needed to fully describe nature.

To accomplish the important goal above, precise measurements of various neutrino oscillation parameters, such as the mixing angles and mass square differences, to precisely determine the hierarchy between the three mass eigenstates and to determine the CP phase $\left(\delta_{\mathrm{CP}}\right)$ are essential. In addition, the detailed study of the $\mathrm{CP}$ violation in the neutrino sector in which the questions such as the neutrino and anti-neutrino behaviors in flavor oscillations. These could lead to a new symmetry through the tension between theoretical predictions and experimental measurements.

In addition to the bread-and-butter physics topic describe above, there are the lingering questions of the grand unification, the energy scale of the unification and the discovery of proton decay which had been sought for a long time. Understanding neutrinos of astrophysical origin, neutrinos from supernova burst and relic neutrinos as well as dark matter and other unknown particles. Especially understanding how the supernova and the black hole can get formed, by looking at the electro-neutrinos coming from supernova explosion would be really important thing for us to learn.

Precision measurements above, require high statistic samples. Since neutrinos interact
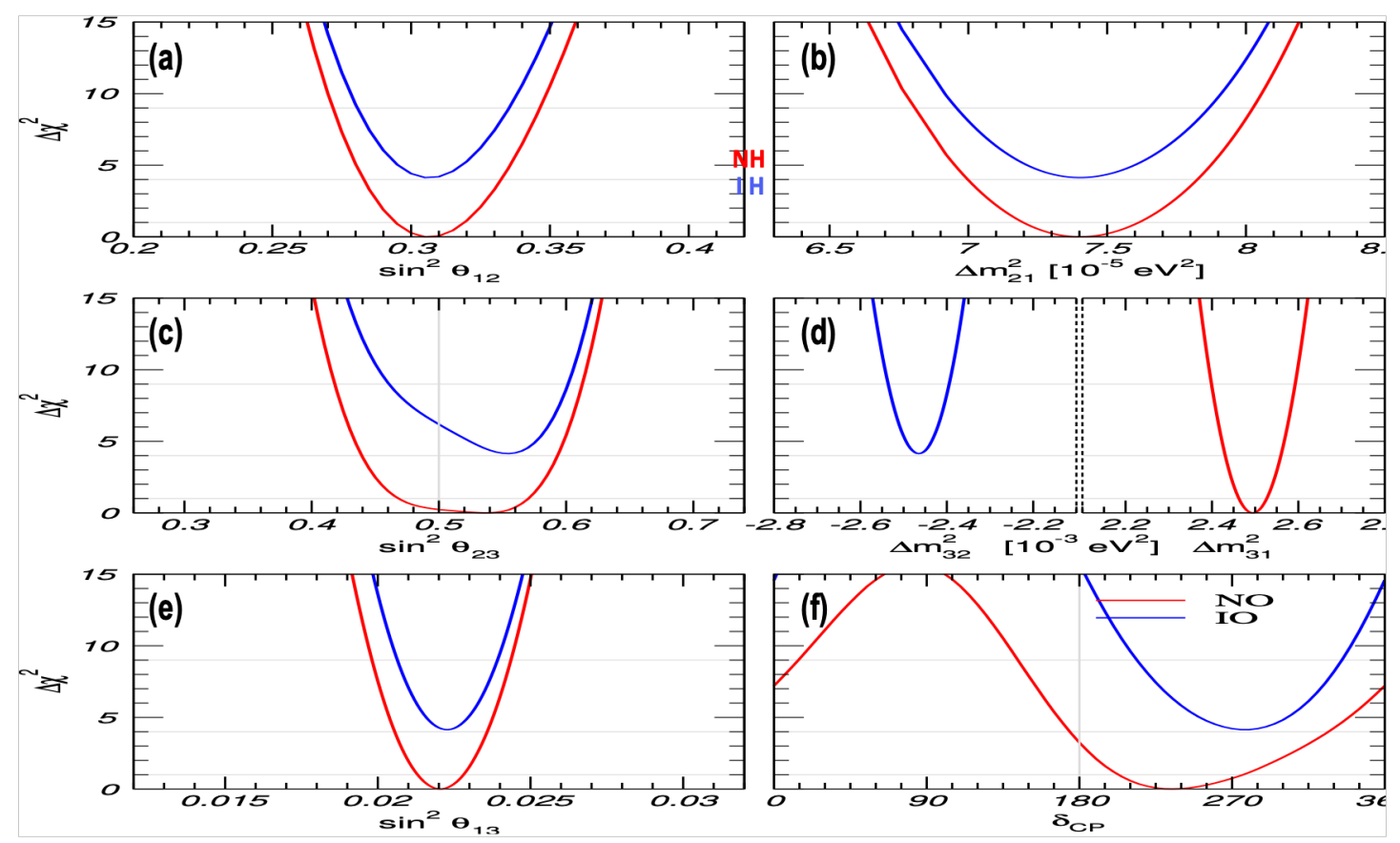

Figure. 1 Neutrino energy spectra measured in DUNE far detector through charged current interactions of (a) electron neutrinos, (b) electron anti-neutrinos, (c) muon neutrinos and (d) muon anti-neutrinos in normal mass hierarchy 
weakly, the experiments require high intensity neutrino beams as well as a large detector mass to obtain sufficient statistics. In addition, to help with reducing systematic uncertainties, the experiments highly capable near and far detector with a long baseline.

\section{Current Status of Neutrino Oscillation Parameter Measurements}

Many experiments have performed measurements of neutrino oscillation parameters. Figure 1 shows the current status of neutrino oscillation parameter measurements based on all available experimental data [5]. Figure 1(a) and a(b) show the $\sin ^{2} \theta_{12}$ and $\Delta \mathrm{M}_{23}^{2}$ measurements from atmospheric neutrino measurements of Super-Kamiokande (SK), IceCube, Km2NET, etc as well as long baseline experiment such as K2K, MINOS, Opera, NOvA, etc.. Figure 1(c) and 1(d) show the $\sin ^{2} \theta_{23}$ and $\Delta \mathrm{M}_{12}^{2}$ measurements from solar neutrino measurements of SNO, SK, Borexino, etc and reactor result from KamLAND experiment.

Finally, Fig.1(e) and 1(f) are $\sin ^{2} \theta_{13}$ and $\delta_{\mathrm{CP}}$ measurement from long baseline experiments, such as MINOS, T2K, NOva, etc and reactor experiments such as Daya Bay, RENO and Double Chooz. The red lines in the plots show the normal hierarchy $(\mathrm{NH})$ while the blue lines show the inverted hierarchy $(\mathrm{IH})$. Precision measurements will narrow the width of these curves and help determine even mass hierarchy.

\section{The Next Generation Neutrino Experiments}

High Energy Physics (HEP) community strategic planning documents in three regions, Europe, US and Japan, have concluded that precise understanding of neutrino oscillation is one of the top priorities. Following this strategy, the efforts of building two next generation neutrino experiments, Deep Underground Neutrino Experiment (DUNE)[6] in the US and the Tokai-toHyper Kamiokande (T2HK) [7] experiments in Japan are in progress in earnest.

\subsection{Hyper Kamiokande, T2HK and T2HKK}

Hyper Kamiokande detector is a water Cerenkov detector and is the far detector of the T2HK and T2HKK experiments. It is expected to be staged in two phases with the active mass of $187 \mathrm{kt}$ of

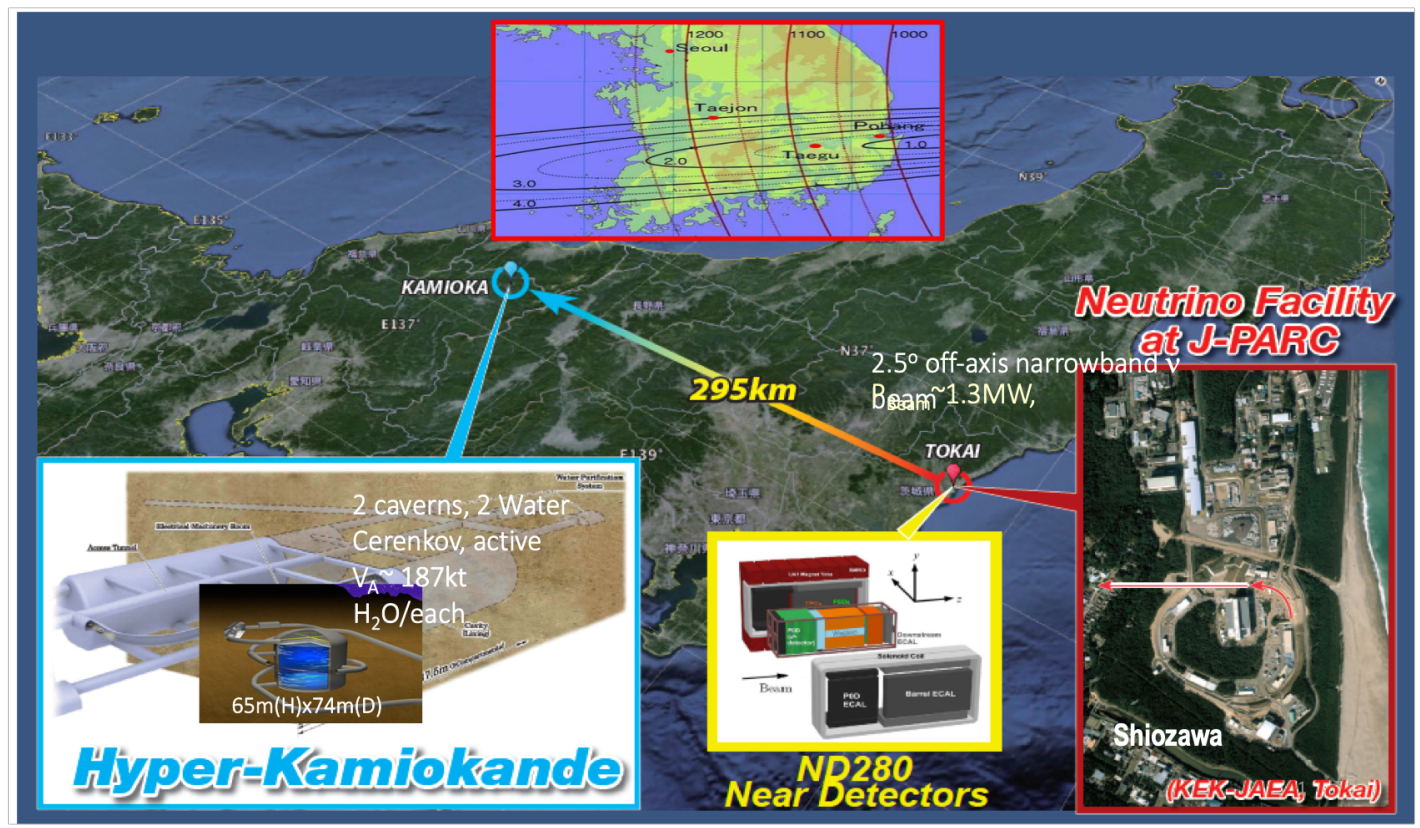

Figure. 2 Hyper-Kamiokande, T2HK and T2HKK schematic diagram. The inset shows the range of neutrino energy spectrum available at different locations in Korea depending on the location of the second detector of $187 \mathrm{kt}$ active mass for T2HKK experiment. The near detector of the experiment is the existing ND280 located $280 \mathrm{~m}$ downstream of the Tokai neutrino beam target, which currently serves as the near detector of the T2K and T2SK experiments. 
water in each detector, about 8 times the mass of the existing Super-Kamiokande (SK) detector at the bottom of Mt. Nijyugo-yama which provides about $650 \mathrm{~m}$ overburden. The dimension of each of the detectors is $65 \mathrm{~m}(\mathrm{H}) \times 74 \mathrm{~m}(\mathrm{D})$. The Hyper-K proto-collaboration consists of about 300 members of 74 institutions from 14 countries. It is supported at the level of 10 million Japanese Yen in fiscal year 2018 by the Japanese government for some basic studies for the experiment. The proto-collaboration has recently released a design report [8] in May 2018.

Figure 2 shows the schematic view of the T2HK experiment for which the beam comes from J-Park facility with high power proton beams. HK detector is located at $2.5^{\circ}$ off-axis, therefore the neutrino beam has narrow energy band for precision measurement. The expected beam-power at the time of running in 2026 is $1.3 \mathrm{MW}$ of $30 \mathrm{GeV}$ proton beams. The neutrino beam for T2HK is shot from Tokai to Kamioka making it a $295 \mathrm{~km}$ long baseline neutrino experiment. Two caverns will be constructed to host the two staged $187 \mathrm{kt}$ active mass water Cerenkov detectors. The near detector of the experiment is the existing ND280 detector located at $280 \mathrm{~m}$ downstream of the Tokai neutrino beam target, which currently is serving as the near detector for the existing long baseline experiment, T2K and T2SK.

T2HKK is an opportunistic experiment which utilizes the neutrino beam that will continue onto Korea. T2HKK expects one 187kt water Cerenkov detector in Kamioka while the second identical $187 \mathrm{kt}$ water Cerenkov detector in one of the two locations in Korea which could provide different baseline, $1000 \mathrm{~km}$ or $1200 \mathrm{~km}$ from Tokai, and provide different neutrino beam energy ranges because of the off-axis angle.

\subsection{Deep Underground Neutrino Experiment}

DUNE (Deep Underground Neutrino Experiment) utilizes the high intensity neutrino beam sent from Fermi National Accelerator Laboratory (Fermilab) in Illinois, US, to the Sanford Underground Research Facility (SURF) in South Dakota, 1300km away from the beam, 1500m underground. DUNE is a joint effort of teams from all three regions, Europe, America and Asia. The collaboration consists of 1132 members of 79 institutions from 32 countries.

DUNE far detector is a liquid argon (LAr) time projection chambers (TPC) of 40kt total

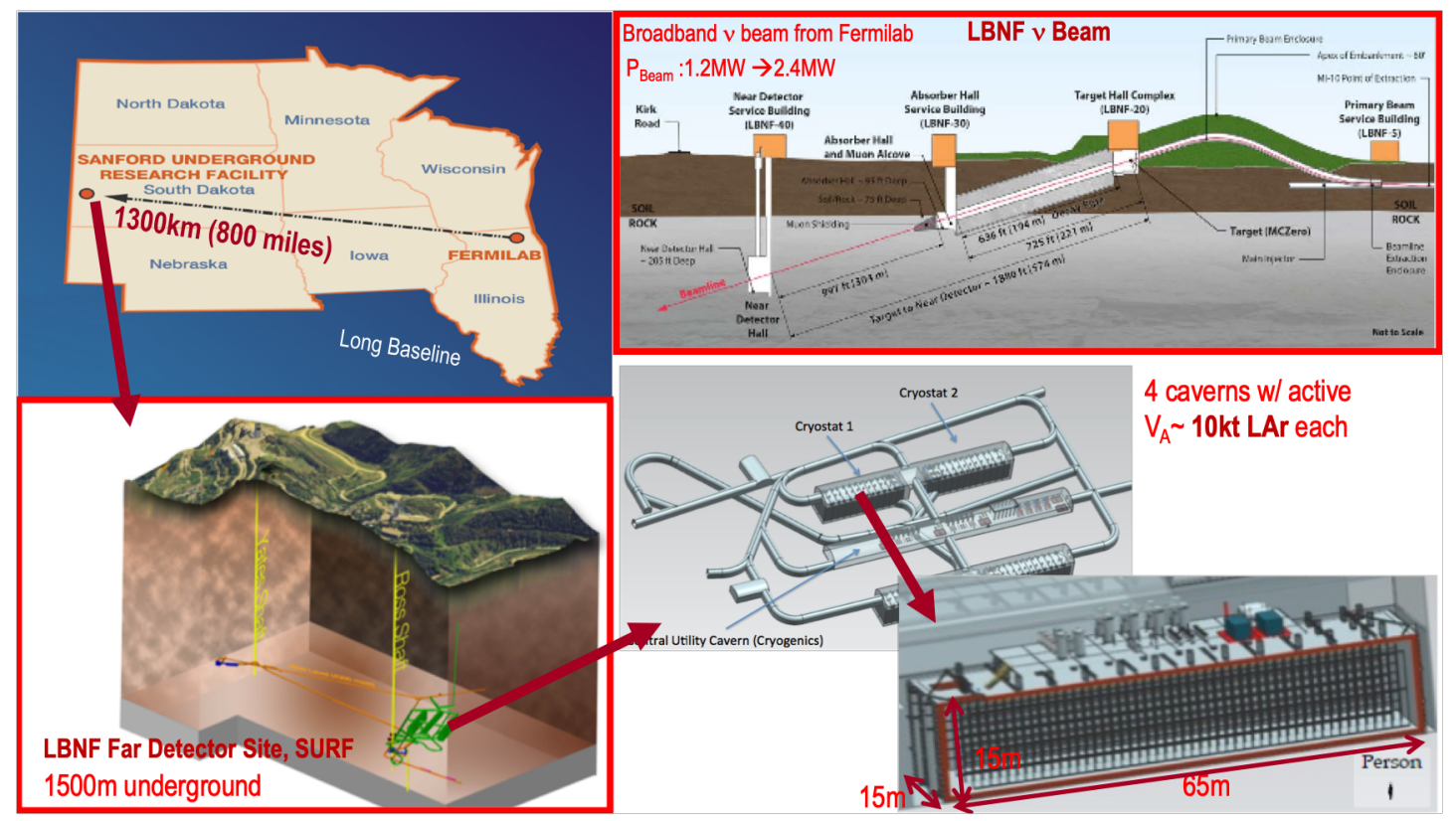

Figure. 3 Schematic diagrams of various component of DUNE. Top right shows the LBNF beam facility at Fermilab, top left shows the relative locations of the beam and the far site, bottom left shows the Sanford Underground Research Facility in Lead, SD, USA 1500m underground and bottom right shows the new area under excavation for the construction of the four caverns and a schematic diagram of one of the four 10kt LAr TPC detectors 
active mass in a staged time line, two $10 \mathrm{kt}$ detectors first followed by the third 10kt about a year after start of the data taking for the first two and the forth $10 \mathrm{kt}$ detector in a couple of years after the third. The first two 10kt detectors will employ two different LAr TPC technologies, the single phase (SP) and the duel phase (DP). Two different technologies within one experiment enables the possibility of a systematic cross-check. As any long baseline neutrino experiment is, DUNE has two components, the Long Baseline Neutrino Facility (LBNF) which consists of beam facility at Fermilab whose schematics is shown in top right of Fig. 3 and the far detector facility at SURF in South Dakota, USA, whose schematics is shown in top left, bottom left and bottom right of Fig. 3.

LBNF beam facility is designed to provide a broadband neutrino beam with the beampower on day one expected to be $1.2 \mathrm{MW}$ and upgradable to $2.4 \mathrm{MW}$ proton beams whose energy is tunable in $60-120 \mathrm{GeV}$. Both the near and far detectors of DUNE are going to be on-axis to take advantage of the magnetic horn focused broadband neutrino beam optimized for ChargeParity Violation studies by enabling access to two oscillation maxima.

The far detector site is $1500 \mathrm{~m}$ underground in a gold mine. U.S. Department of Energy has approved the construction of four new caverns that will host four LAr TPC detectors, each of which has 10kt active mass as shown in the bottom right figure of Fig. 3. Each of the four cryostats will have inner dimension of $15 \mathrm{~m}(\mathrm{~W})$ X $15 \mathrm{~m}(\mathrm{H})$ X $65 \mathrm{~m}(\mathrm{~L})$, about the size of a large building. And going directly to this kind of thing is not that easy, because there are a lot of things that we have to do, like scalability and quality control and quality insurance and stuff like that.

Table 1 below summarizes the design parameters. As can be clearly seen, the capability of the beam facility and the detectors are complimentary to each other for precision studies of the neutrino oscillation properties. And Hyper-K actually necessarily has T2HK and T2HKK in it. DUNE is a liquid argon TPC. We're actually planning on going two detectors first, with the $10 \mathrm{kt}$ active volume followed by two detectors one first and the forth a couple of years later. For the case of the T2HK, the table shows the staged approach in which one $187 \mathrm{kt}$ detector is built first followed by the second $187 \mathrm{kt}$ detector. T2HKK, on the other hand, has $187 \mathrm{kt}$ detector in Kamioka and another $187 \mathrm{kt}$ detector in Korea.

\subsection{Financial Support}

For large scale experiments like T2HK and DUNE, securing financial support is an essential aspect of the project. Hyper-K experiment was chosen as one of the 14 top priority science projects selected by the Japanese government (MEXT) in 2017. As a result, the experiment has been supported 10 million Japanese Yen in fiscal year 2018. While this amount does not represent a large support, the fact that the project was supported at all means a lot more than what amounts it's telling you, since it implicates that the experiment can be approved this year. An important government decision is expected before the end of 2018 since the input to European strategy document is by December 2018.

Table 1. Summary of DUNE, T2HK and T2HKK design parameters

\begin{tabular}{|c|c|c|c|}
\hline & DUNE & HK/T2HK & T2HKK \\
\hline $\begin{array}{c}\text { FD Technology \& } \\
\text { Fiducial Mass }\end{array}$ & $\begin{array}{c}\text { LArTPC } \\
(2+1+1) \times 10 k t\end{array}$ & $\begin{array}{c}\text { Water Cerenkov } \\
(1+1) \times 187 \mathrm{kt}\end{array}$ & $\begin{array}{c}\text { Water Cerenkov } \\
187 \mathrm{kt}(\mathrm{Kamioka}) \\
187 \mathrm{kt} \text { (Korea) }\end{array}$ \\
\hline Proton Beam power & $1.2 \mathrm{MW} \rightarrow 2.4 \mathrm{MW}$ & \multicolumn{2}{|c|}{$1.3 \mathrm{MW}$} \\
\hline Proton Energy (GeV) & $60-120$ & \multicolumn{2}{|c|}{30} \\
\hline Baseline $(\mathrm{km})$ & 1300 & 297 & 1100 \\
\hline FD Depth $(\mathrm{m})$ & 1500 & 650 & 1120 \\
\hline ND from $v$ target $(\mathrm{m})$ & 575 & \multicolumn{2}{|c|}{280} \\
\hline
\end{tabular}


DUNE, on the other hand, is going slightly differently. As was pointed out in previous sections, the cavern construction at the has been fully approved by the U.S. Department of Energy, the hosting agency and the excavation process has begun with a support over $\$ 100 \mathrm{M}$ level. And then support for LBNF neutrino beam facility which covers the proton beam, the neutrino target, the secondary beam focus, decay chain for neutrino beam generation and the beam dump is expected in a couple of years. Independently, the proton improvement plan necessary for high intensity proton beam for $1.2 \mathrm{MW}$ beam power is under consideration separately from LBNF by the U.S. government.

Detector construction for prototyping also is in progress at CERN. CERN is an essential partner in DUNE. It has constructed neutrino platform in the north area of CERN beam test area in Previssin site on French side. It has built two cryostats for ProtoDUNE and is committed to build the cryostat for the first DUNE detector of the four. At the time of this presentation, the first meeting with the GTT the company that builds these cryostats occurred, to discuss about design parameters. CERN is playing a leadership role in installation of prototypes and the operation of prototype.

Finally, U.K. has agreed in September 2017, to award \$88M project grant for UK institutions on DUNE. In addition, many other countries, such as France, Switzerland, Italy, Brazil, Korea, etc are making various contributions at different stages of the experiment. The commitment of the support DUNE currently has garnered is several hundred million dollars.

\section{Physics Reach}

With the capacities of DUNE and T2HK experiments provided in the previous section, what would be the expected physics reach? The plots in Fig. 4 show the neutrino energy spectrum measured through their charged-current interactions at DUNE far detector. Figure 4. (a) and (c) are the spectrum of the electron neutrinos resulting from muon neutrino oscillation and that of the muon neutrinos which clearly show depression at the peak of the electron neutrino spectrum from
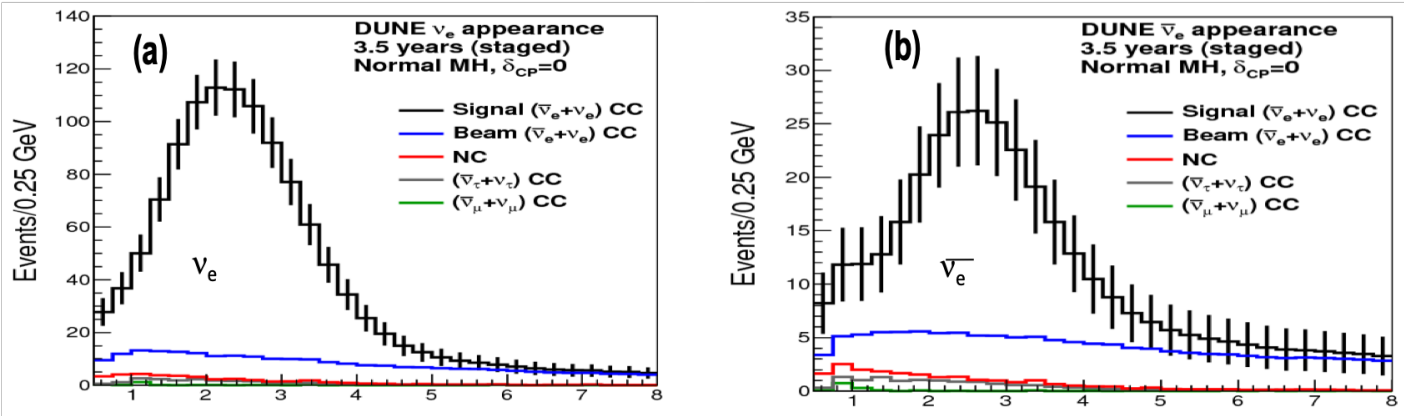

Reconstructed Energy (GeV)
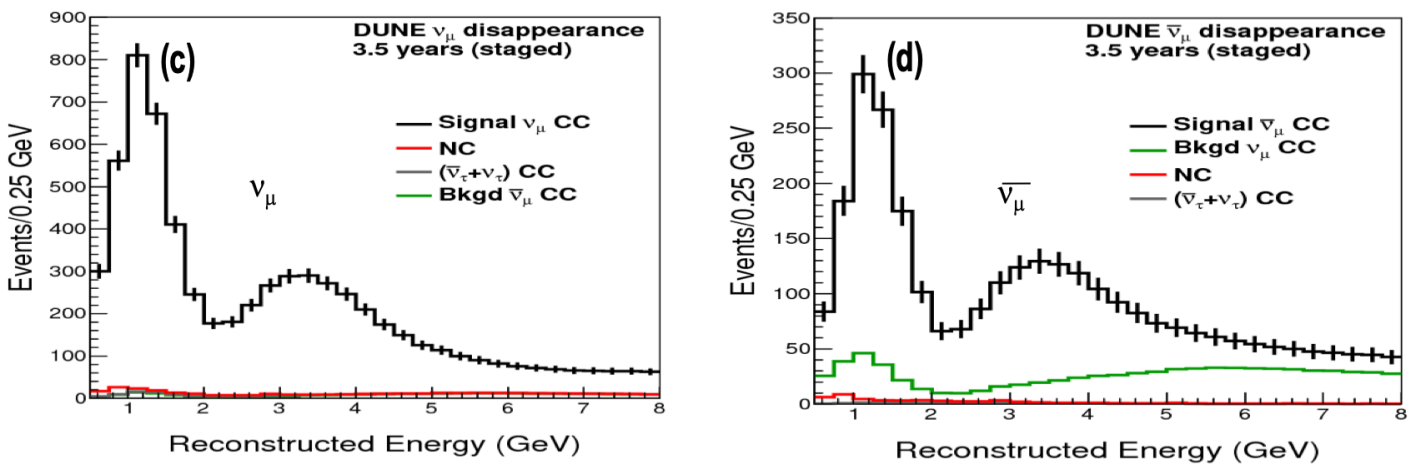

Figure. 4 Neutrino energy spectra measured in DUNE far detector through charged current interactions of (a) electron neutrinos, (b) electron anti-neutrinos, (c) muon neutrinos and (d) muon anti-neutrinos in normal mass hierarchy 
the disappearance of them in neutrino mode, respectively. Similarly, Figure 4. (b) and (d) show the corresponding spectra in anti-neutrino running mode in 3.5 year running each of neutrino and anti-neutrino modes for a staged scenario for the 70kt-year of data collection [9].

By comparing the measured neutrino flux in the far detector in either the depression or appearance in these neutrino energy spectra to the un-oscillated neutrino flux, the oscillation parameters can be measured precisely. The higher the statistics the more precise the measurements become in terms of its statistical uncertainties. In order to obtain more precise measurements beyond that of the statistical uncertainly, however, it is critical to have a good systematic understanding of the neutrino flux before the oscillation. The un-oscillated neutrino flux can be determined by very good near detectors. It, therefore, is very important for us to have near detector that are capable of measuring the flux precisely before any oscillation.

The plots in Fig. 5 show the sensitivity for determining CP phase $\left(\delta_{\mathrm{CP}}\right)(\mathrm{a})$ for Hyper-K with one $187 \mathrm{kt}$ active mass for 10 year running and (b) for DUNE with staged approach for two $10 \mathrm{kt}$ active mass followed by additional two with a few year separation in between for 7 year (green) and for 10 year (orange) running in normal mass ordering scenario. The band in Fig.5(b) reflects the uncertainties in $\theta_{13}$ and $\theta_{23}$ measurements [5] as indicated in the figure. The two dashed horizontal lines in both the plots show $3 \sigma$ and $5 \sigma$ significance. These show that a wide range of $\delta_{\mathrm{CP}}$ can be covered in each of the experiments with the potential for discovery beyond $5 \sigma$ level in about $0.2 \pi<\delta_{\mathrm{CP}}<0.8 \pi$ range. It important to point out that expected resolution of $\delta_{\mathrm{CP}}$ is of the order 10 degrees at $\delta_{\mathrm{CP}}=0$ for DUNE.
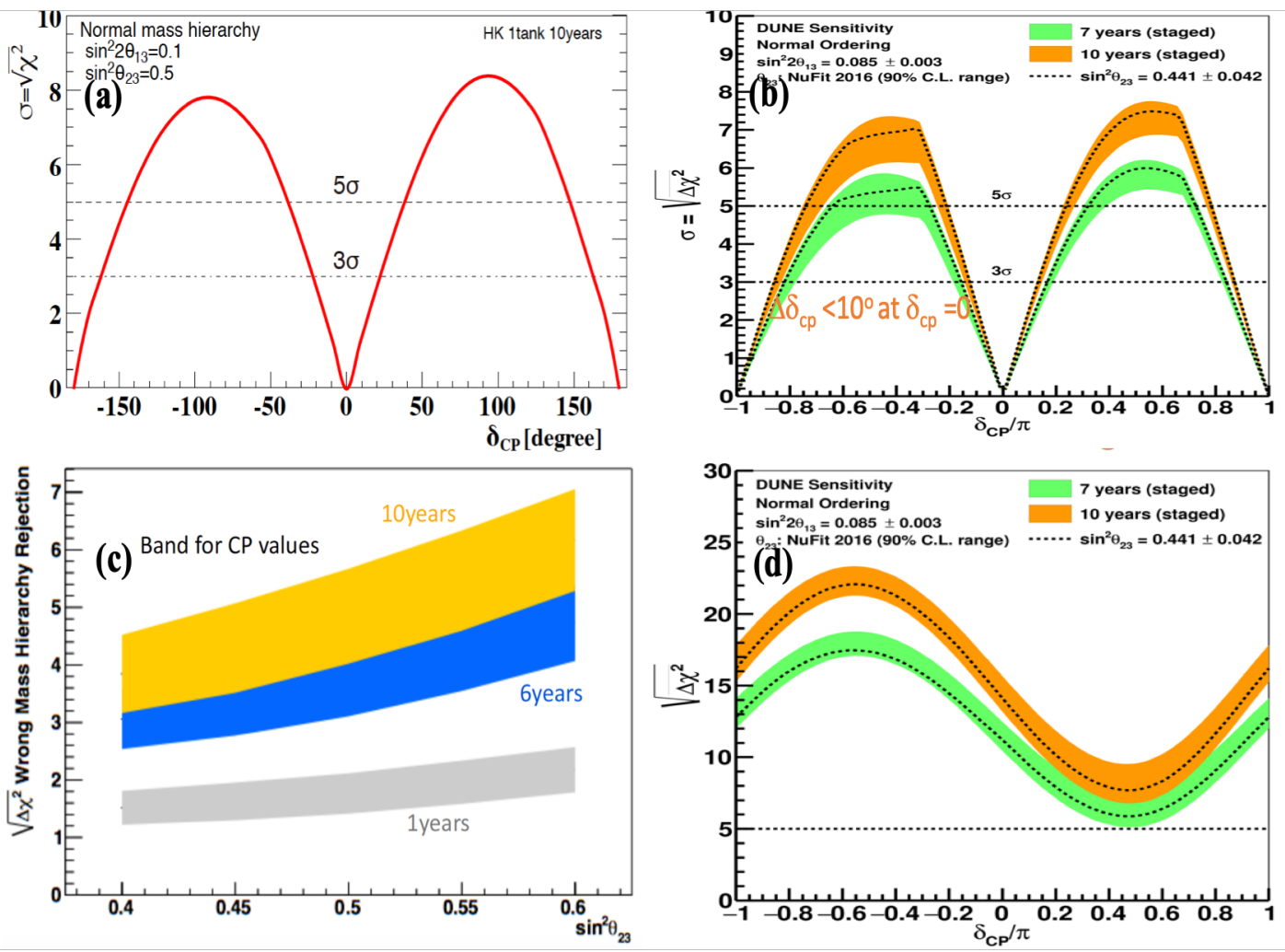

Figure. 5 Exclusion plots for $\delta_{\mathrm{CP}}$ (a) for Hyper-K experiment with 10 year running of one $187 \mathrm{kt}$ detector and (b) for DUNE in 7 year (green) and 10 year (orange) data taking with a staged active mass scenario and the significance in mass ordering determination (c) for Hyper-K as a function of $\sin ^{2} \theta_{23}$ with 10 year running of one $187 \mathrm{kt}$ detector and (d) for DUNE as a function of $\delta_{\mathrm{CP}}$ in 7 year (green) and 10 year (orange) data taking with a staged active mass scenario 
Figure 5.(c) shows the exclusion for mass ordering in Hyper-K detector in a one year (grey band) versus six (blue band) to ten year (yellow band) data taking, clearly demonstrating over $3 \sigma$ level rejection in the entire range of $\sin ^{2} \theta_{23}$ after 10 years of data taking with one $187 \mathrm{kt}$ detector. Figure 5.(d) shows the significance of mass ordering determination as a function of $\delta_{\mathrm{CP}}$ with staged approach for two 10kt active mass followed by additional two with a few year separation in between for 7 year (green) and for 10 year (orange) running, clearly demonstrating over $5 \sigma$ level significance of mass ordering determination for all range of $\delta_{\mathrm{CP}}$ after 7 years of data taking.

More exciting potential is the possibility for observing supernova burst. It is expected that when a supernova explosion happens, the flux of the electron-neutrinos generated from the burst depends on the elapsed time from the time of burst. Figure 6 shows the measured electron as a function of time from the burst in seconds (a) in Hyper-K and (b) in DUNE far detector through the reaction $v_{e}+{ }^{40} \mathrm{Ar} \rightarrow e^{-}+{ }^{40} K^{*}$. As can be seen in Fig.6(a) and (b), as a function of time from the burst of the supernova, the time dependence of the electron neutrino flux differs depending on the process of the burst and collapse into a black hole, especially in $0-0.25$ seconds after the burst, enabling the experiment to explore in detail the model for black hole formation in real time. In addition, as Fig. 6(b) shows, the electron neutrino flux in $0.02-0.05$ second range differ dramatically between no-oscillation (blue), oscillation with the normal mass ordering (red) and oscillation with the inverted mass ordering (green). Therefore, precise measurement of electron neutrino flux as a function of elapsed time from the supernova burst enables clear determination of mass ordering.

Finally, thanks to high intensity proton beams and the large mass highly capable detectors, physics beyond the Standard Model (BSM) can be pursed in these future neutrino experiments. In particular, since DUNE is LAr TPC detector that can provide precision measurements of timing, energy and tracks together with low energy detection threshold, many BSM topics can be explored in the experiment. DUNE can perform searches for low-mass dark matter created in the beam and the boosted dark matter of cosmogenic origin. LDM could be produced in the neutrino target through a kinematic coupling [10] and detected in DUNE via elastic scattering resulting in a knockoff electron or a nuclear recoil, just like neutrinos. Since LDM particles are expected to be heavier than neutrinos, their average arrival time to the near detector is later than that of the neutrinos, providing a discriminating tool. In addition, many other BSM topics, such as searches
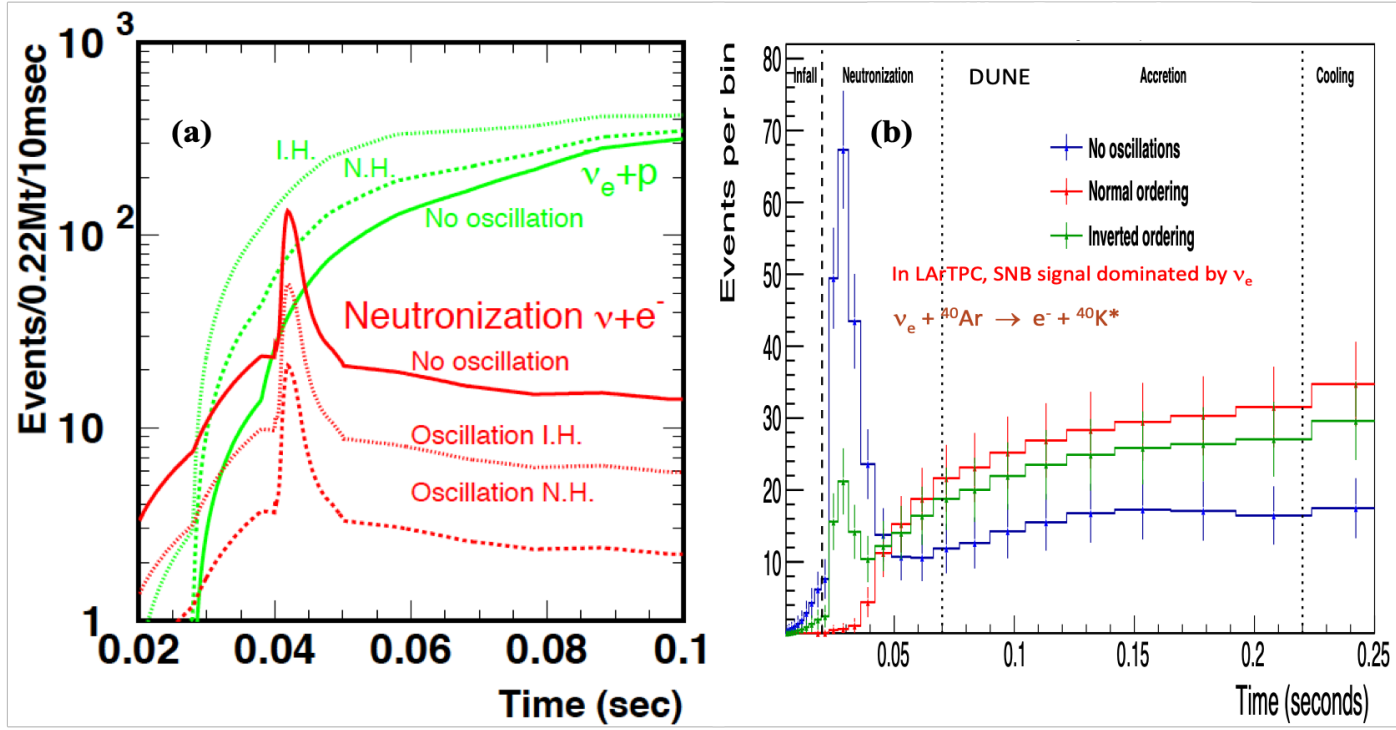

Figure. 6 Electron neutrino flux as a function of time in seconds from the super-nova burst (a) for Hyper-K and (b) DUNE far detector for three different mass ordering scenarios 
for sterile neutrinos, non-standard interactions, boosted dark matter and heavy neutral leptons are feasible. An interesting opportunity for early physics at DUNE is searching for booted dark matter in its inelastic scattering using ProtoDUNE detectors [12]. The BSM opportunities are the area of physics which promotes the collaboration between experimentalists and phenomenologists.

\section{Prototype Detectors and Experiment Timelines}

Since it is necessary to construct prototypes prior to building large-scale experiments like DUNE and HK, DUNE has been building two prototype detectors at CERN neutrino platform, one each for single and dual-phased technologies. In a single-phase detector, the interaction occurs inside the liquid, and the ionization electrons from the traversing charged particles resulting from the interaction drift horizontally and get detected by the anode wire plane in liquid. The active volume of the single phase ProtoDUNE detector (ProtoDUNE SP) is $6.9 \mathrm{~m}(\mathrm{~W}) \mathrm{X} 7.2 \mathrm{~m}(\mathrm{~L}) \mathrm{X} 6 \mathrm{~m}(\mathrm{H})$ split into two $6.9 \mathrm{mX} 3.6 \mathrm{~m}$ modules that share the single cathode plane. In the dual phase ProtoDUNE (ProtoDUNE DP), the interaction occurs in the liquid argon but the ionization electrons drift vertically upward to the liquid-gas interface, extracted from liquid into the gas and amplified through the Large Electron Multiplier (LEM) before the charges get collected in gas. The active volume of ProtoDUNE DP is $6 \mathrm{~m}(\mathrm{~W}) \mathrm{X} 6 \mathrm{~m}(\mathrm{~L}) \mathrm{X} 6 \mathrm{~m}(\mathrm{H})$.

At the time of this presentation, ProtoDUNE SP was undergoing a leak check which was then followed by the gas purge in preparation for liquid fill. Proto-DUNE DP field cage was completed and has been tested at $150 \mathrm{kV}$ in air. Using a smart wiring the testing was carried out at the operational voltage differentials between each of the field shaping rings at $3 \mathrm{kV}$. The important fact in these prototypes is that most of the components used in construction are the same size to be used in DUNE the final detectors, so ProtoDUNE detectors are almost completely scalable to the final DUNE detector.

As summarized in table 2, DUNE cavern excavation on going and will be completed by 2020 while Hyper-K is to begin 2019 and ends on 2024. The Hyper-K cavern excavation, however, hinges on the approval of the construction by the Japanese government in 2018. As pointed out in previous section, the Hyper-K proto-collaboration has completed and released its design report in May 2018, while DUNE has completed its conceptual design report [9] in 2015 and followed it up with a more detailed interim design report [11] in April 2018.

The construction of DUNE far detector is expected to be in $2022-2024$ for two 10kt active mass detectors, following the completion of the cavern excavation and the construction of the two cryostats. DUNE far detector cryostats are scheduled to be filled with LAr in $2024-$ 2025 , enabling the start of cosmic ray data taking in 2025. The beam will become available with the $1.2 \mathrm{MW}$ power in 2026 . The construction of the Hyper-K detector with one $187 \mathrm{kt}$ active mass is anticipated in $2024-2025$, followed by the water fill in $2025-2026$ with the neutrino beam

Table 2. Timeline of DUNE and Hyper-K Experiment

\begin{tabular}{|c|c|c|}
\hline & DUNE & Hyper-K \\
\hline Cavern Excavation & $2018-2020$ & $2019-2024$ \\
\hline FD Construction & $2022-2024$ & $2024-2025$ \\
\hline FD Fill & $\begin{array}{c}2024-2025 \\
\left(10+10 \mathrm{kt} \mathrm{V}_{\mathrm{A}}\right)\end{array}$ & $\begin{array}{c}2025-2026 \\
\left(187 \mathrm{kt} \mathrm{V}_{\mathrm{A}}\right)\end{array}$ \\
\hline Data Taking & $\begin{array}{c}2025(\text { cosmic }) / \\
2026(\mathrm{n} \text { beam) }\end{array}$ & 2026 \\
\hline ND Ready & 2027 & In place \\
\hline
\end{tabular}


from 1.3MW protons become available in 2026.

Finally, the near detector for DUNE which will be located about $570 \mathrm{~m}$ downstream of the neutrino target will have its conceptual design report completed late 2019 in time for the technical design report of DUNE far detector and will be ready for data taking in 2027. Hyper-K near detector, however, is already in place since it will be using the ND280 which is already serving T2K and T2SK experiments.

\section{Conclusions}

The neutrino sector and the Standard Model needs to be modified thanks to the solid experimental proof of neutrino flavor oscillation. Precise understanding of neutrino oscillation phenomena, precision measurements of the oscillation parameters and determining CP violation phase in lepton sector is essential. Two complimentary, next-generation long baseline neutrino experiments with large neutrino target mass and high intensity neutrino beams, DUNE and HK are in preparation.

Hyper-K experiment is recognized by the Japanese government as one of the seven priority science projects, selected every four years by MEXT, and is eagerly awaiting Japanese government approval. DUNE has been garnering strong financial supports and commitments from U.K., U.S.A., CERN and other partner countries. The far site construction in South Dakota is fully funded and began in 2017 with the ground breaking in July 2017. Two large-scalable, DUNE prototype detectors are to start taking data in 2018 (as of September 21, 2018, the single phase ProtoDUNE detector started taking data). The construction schedules for both DUNE and HK experiments target beam data in 2026.

Finally, it would be great achievement that in the next quarter century $\mathrm{CP}$ violation in neutrino sector or the lepton sector is discovered, a new theoretical framework which can replace the Standard Model or can enhance the Standard Model become available, supersymmetric particles are discovered at the Large Hadron Collider and the dark matter is observed. The largemass detectors necessary for precision neutrino experiment will enable accomplishing three of these four visions.

\section{References}

[1] S. L. Glashow, Nucl. Phys. 22, 579 (1961); S. Weinberg, Phys. Rev. Lett. 19, 1264 (1967); A. Salam, "Weak and electromagnetic interactions," in Elementary particle physics: relativistic groups and analyticity, N. Svartholm, ed., p. 367. Almqvist \& Wiskell, Proceedings of the eighth Nobel symposium (1968)

[2] F. Abe et al, CDF Collaboration, Phys. Rev. Lett. 74, 2626 (1995); S. Abachi et al., DZero Collaboration, Phys, Rev. Lett. 74, 2422 (1995)

[3] ATLAS Collaboration, Phys. Lett. B716, 1 (2012); CMS Collaboration, Phys. Lett. B716, 30 (2012)

[4] V.Barger, D.Marfatia, K.L.Whinsnant, "The Physics of Neutrinos," Princeton University Press,. ISBN 978-0-691-12853-5 (2012)

[5] I.Esteban, M.C. Gonzalez-Farcia, A.Hernandez-Cabezudo, M.Maltoni, T.Schwetz, arXiv:1811.05487 (2018) NuFit3.2

[6] Deep Underground Neutrino Experiment, http://www.dunescience.org/

[7] Hyper-Kamiokande Collaboration, http://www.hyperk.org/

[8] Hyper-K design report, arXiv:1805.04163 (2018)

[9] DUNE Conceptual Design Report (CDR), arXiv:1512.06148 (2015)

[10] B. Batell, M. Pospelov, and A. Ritz, Phys.Rev. D80, 095024 (2009)

[11] DUNE Collaboration, DUNE Far Detector Interim Design Report, arXiv:1807.10334 (volume 1), arXiv:1807.10327 (volume 2), and arXiv:1807.10340 (volume 3) (2018)

[12] A.Chatterjee at al., "Searching for Boosted Dark Matter at ProtoDUNE," Phys. Rev. D98, 075027 (2018) 